\title{
Estimation of Structural Reliability of Lifting System for Jacket-Up Barge using Monte Carlo Simulation
}

\author{
Chang-Yong Song ${ }^{1}$, Woo-Chang Park ${ }^{1}$, Jung-Wook Yang ${ }^{1}$, Seong-Ki Hong ${ }^{1}$, Jonghwan Park ${ }^{1}$, Mun Yong \\ Kim $^{2}$, Jinseo Kang ${ }^{2}$ \\ ${ }^{1}$ Dept. of Naval Architecture \& Ocean Engineering, Mokpo National University \\ 58554, Jeonnam, South Korea \\ cysong@mokpo.ac.kr; heavenly-sea@hanmail.net; didwjddnr12@naver.com; skhong327@mokpo.ac.kr; \\ jongpark@mokpo.ac.kr \\ ${ }^{2}$ Myung Il Co., Ltd. \\ 54002, Jeonbuk, South Korea \\ mipmkmy@gmail.com; jinseo82@hanmail.net
}

\section{Extended Abstract}

Jack-up barge, one of the representative special vessels for installation and maintenance of offshore structures such as offshore plants, offshore facilities, and offshore renewable energy systems, secures the work stability of barge using mechanical holding force developed from leg or spud which is mounted on seabed. Lifting system for jacket-up barge is the key equipment to maintain the holding force as well as to mount and to move the spud on the seabed under an extreme marine environment [1]. Typical lifting system types for offshore installation and maintenance are known as hydraulic pin type and rack-pinion.

Recently, a new type of structural design has been devised to improve the holding power of lifting systems. Design uncertainty can exist in case that the evaluation of structural performance of such new type of lifting system is carried out only by standard design guidance such as classification rules.

In this study, reliability analysis was performed for a new concept multi-ball holding clamp type lifting system with 900 ton class holding capacity which was developed to improve the holding power. In the context of reliability analysis, Monte Carlo Simulation (MCS) based on the variance reduction method was applied to quantitatively evaluate the uncertainties in the structural performance evaluation $[2,3]$. The evaluation of the structural strength performance was carried out by using the loading conditions which combine the mechanical loads of the operating cylinders with design load conditions for the deck lifting device of the mobile offshore structure specified by the classification rule [4]. In order to perform the MCSbased reliability evaluation of the lifting system, a three-dimensional finite element analysis (FEA) model was generated, and the analysis results of the structural stress and deformation were compared to the allowable stresses recommended by the classification rule. The structural analysis results were used to evaluate the reliability of the structural performance considering uncertainties on design, manufacturing, and loading. From the reliability analysis, the lifting system design was determined satisfying a target reliability index.

\section{Acknowledgments}

This research was supported by MOTIE \& KIAT through the Promoting Regional Specialized Industry.

\section{References}

[1] American Bureau of Shipping, "Marine Operation Manual for 90m Self-Elevating Unit," ABS Technical Report, 2016.

[2] M. Papadrakakis and N. D. Lagaros, "Reliability-based structural optimization using neural networks and Monte Carlo simulation," Comput Methods Appl Mech Eng., vol. 191, no. 32, pp. 3491-3507, 2002.

[3] C. Y. Song and J. Lee, "Reliability-based design optimization of knuckle component using conservative method of moving least squares meta-models," Probabilistic Eng. Mech., vol. 26, no. 2, pp. 364-379, 2011.

[4] Det Norske Veritas, "DNV-RP-C104 - Self-elevating Units," DNV Recommended Practice, 2012. 